Journal of the Operations Research

Society of Japan

Vol. 42, No. 2, June 1999

\title{
THE HYPERCORE ON SOCIAL CHOICE PROBLEM
}

\author{
Hisakazu Nishino \\ Keio University
}

\author{
Wentian Cui \\ Toyohashi University \\ of Technology
}

\author{
Masayoshi Mizutani \\ Tokyo Keizai University
}

\author{
Yuuji Satoh \\ Matsusaka University
}

(Received August 29, 1997; Final February 22, 1999)

\begin{abstract}
In this paper, a class of stable and coalitionally nonmanipulable social choice correspondences is presented. Each correspondence in this class, called the hypercore, is induced from a social choice function with restricted domain of preference profile. It is proved that the correspondence is the intersection of cores over an equivalent class of profiles. On the contrary to Demange's max-max criterion, maxmin criterion is adopted for defining a coalitional nonmanipulability. Although the core induced from a coalitionally nonmanipulable social choice function with a restricted domain does not necessarily satisfy the nonmanipulability in the max-min sense, it is shown the hypercore does.
\end{abstract}

\section{Introduction}

By a society, we mean a set $N=\{1,2, \cdots, n\}$, consisting of the $n$ agents. It is assumed that each agent $i \in N$ has a linear preference order $\succ_{i}$ over a set of $m$ possible social alternatives, $A=\left\{a_{1}, a_{2}, \ldots, a_{m}\right\}$. Let $\mathcal{L}$ be the set of all linear preference orders on $A$. A preference profile of the society is defined by $\succ_{N}=\left(\succ_{1}, \succ_{2}, \cdots, \succ_{n}\right)$. The set of all profiles is denoted by $\mathcal{L}^{N}$.

The focus of social decision problem initially was to identify a 'rational' rule for combining agents' profiles into an ordering $\succ \in \mathcal{L}$. Formally, a rule is described by a social welfare function, which is a mapping from $\mathcal{L}^{N}$ to $\mathcal{L}$. ARRow [1] showed that there exists no social welfare function satisfying Pareto optimality, independence of irrelevant alternatives and nondictatorialness.

This fact led us to the studies of social choice problem instead of the social welfare problem. In a social choice problem, we seek to identify a rational rule for combining agents' preferences into an alternative $a \in A$. That is, the requirement of finding a linear order: is relaxed. The rule is described by a social choice function (SCF) which is a mapping $h$ from $\mathcal{L}^{N}$ to $A$. However, GibBard-Satterthwaite theorem $[5,8]$ claims that any individually nonmanipulable SCF defined over all possible profiles is either dictatorial or satisfies $\left|h\left(\mathcal{L}^{N}\right)\right| \leqq 2$. This result hinders a naive attempt to find a reasonable solution to the social choice problem.

Several alternative paths have been explored to circumvent the negative results mentioned above. We review some of them here. BARBERA, Sonnens Chein and Zhou [2] considered a problem of the approval voting. Since the approved set of alternatives can take any subset of $A$ including the emptyset, it is necessary to assume that each agent has a linear preference order on the power set $2^{A}$ of $A$. They showed that, when the preference orderings on $2^{A}$ are 'separable', a voting scheme satisfies both individual nonmanipulability and 'sovereignty' if and only if the voting scheme is 'voting by committees'. This result is strong but is only applicable to a specific scheme of approval choice.

Moulin and Peleg [6] explored another path by introducing the effectivity function 
(EF). EF indicates the power of each subset of $N$ on the result of social choice when all agents of the subset cooperate together. They defined the consistency condition that is a relaxation of individual nonmanipulability, and studied the core of EF.

The concept of nonmanipulability employed in the above two approaches is rather weak in the sense that it leaves no room of manipulation only for individuals but is susceptible to coalitional manipulation. Also, note that the result of BARBERA, SonNEnschein and ZHoU [2] holds true under the restriction of domain $\mathcal{L}^{N}$. One promising way to go around these problems appears to be the use of social choice correspondence (SCC), which is a mapping from $\mathcal{L}^{N}$ to $2^{A}$. SCC relaxes the requirement of the definite value property imposed on SCF. Under this setting, DEMANGE [4] introduced optimistic coalitional nonmanipulability. Specifically, SCC is optimistically coalitionally manipulable to $S \subset N$ if the following two conditions are satisfied: (1) All agents in $S$ prefer some alternative to every alternative in the current SCC value. Here the current SCC value is the value of SCC when all agents reveal their true preferences. (2) Agents in $S$ can insert the preferred alternative into the SCC value by presenting their preferences strategically. The qualifier "optimistic" is added to coalitional manipulability since the coalition members are interested only in inserting the preferred alternative into the SCC value, and not in excluding a disliked one. That is, the coalition members optimistically hope that their favorite alternative in SCC would be eventually selected. Demange introduced a condition called strong stability for EF. This condition includes the convexity of EF and further assures the nonemptiness of the core. It was shown that the strong stability of EF implies the optimistically coalitional nonmanipulability of the induced core. Her result has a wide range of applicability since she deals with a general class of SCCs. Unfortunately, no actual example of voting rule which induces her strong stability EF was shown.

The purpose of this paper is to construct a class of SCCs each of which satisfies the stability (nonemptiness) and the coalitional nonmanipulability based on max-min criterion. Though Demange introduced both optimistic and pessimistic coalitional nonmanipulability, the latter of which is similar to our coalitional nonmanipulability in max-min sense, she focused her discussion only on the properties concerning the former. We would like to emphasize that our approach is not based on EF.

Every social choice function (single-valued SCC) defined on the whole $\mathcal{L}^{N}$ is defective with respect to manipulability as stated in GIBBARD-SATTERTHWAITE $[5,8]$. We thus introduce a restricted social choice function (RSCF) $f$ which is a function from a nonempty subset $\mathcal{D}_{f}$ of $\mathcal{L}^{N}$ to $A$ and is nonmanipulable in its domain $\mathcal{D}_{f}$. We construct an SCC by assigning a subset of $A$ to each profile not in the domain of $f$, keeping its stability and coalitional nonmanipulability. The core may seem to be one of reasonable candidates for such SCCs. However, the core induced from $f$ is not necessarily coalitionally nonmanipulable in the max-min sense. We thus propose a new class of coalitionally nonmanipulable SCCs in the max-min sense. Each SCC in this class is induced from RSCF $f$ and is called hypercore $H_{f}$. We derive some useful properties of $H_{f}$. It is shown among others that $H_{f}$ is a subset of the core of $f$ for every profile. Also, if a profile belongs to the domain of $f$, then $H_{f}$ is a singleton consisting only of the value of $f$. We introduced a condition on $f$ called noncovering, which is a sufficient condition for the coalitional nonmanipulability of $f$. In contrast with Demange's strong stability, it is easily shown that a class of restricted majority rules provides a simple example of RSCFs with noncovering property. It is proved that the hypercore $H_{f}$ is stable if and only if $f$ satisfies the noncovering property.

\section{Notation and Basic Concepts}

\subsection{Restricted social choice function and noncovering property}


Let $N=\{1,2, \cdots, n\}$ be a society, the set of agents, $n \geqq 2$, and $A=\left\{a_{1}, a_{2}, \ldots, a_{m}\right\}$ be the set of alternatives, $m \geqq 2$. A coalition is a nonempty subset of $N$. By $2^{A}$ we denote the set of all subsets of $A$, and let $P(A)=2^{A}-\{\emptyset\}$. A preference ordering of each agent $i \in N$, denoted by $\succ_{i}$, is assumed to be a linear ordering on $A$, namely, an irreflexive, transitive and connected binary relation. Let $\mathcal{L}$ be the set of all linear preference orderings on $A$. An $n$-tuple $\succ_{N}=\left(\succ_{1}, \succ_{2}, \ldots, \succ_{n}\right)\left(\succ_{i} \in \mathcal{L}, i \in N\right)$ is called a preference profile, and $\mathcal{L}^{N}$ denotes the set of all possible preference profiles. For any coalition $S, \succ_{S}$ denotes the projection of $\succ_{N}$ over $S$, and $\left(\succ_{N-S}, \succ_{S}^{\prime}\right)$ denotes the profile in which preference of $S$ is replaced by $\succ_{S}^{\prime}$.

Given $\succ_{i} \in \mathcal{L}$ and $B \in P(A)$, let $\bar{a}\left(B ; \succ_{i}\right)\left(\underline{a}\left(B ; \succ_{i}\right)\right)$ denote agent $i$ 's best(worst) alternative among $B$ under $\succ_{i}$, i.e.,

$a \nsucc_{i} \bar{a}\left(B ; \succ_{i}\right)$ for every $a \in B$ and

$\underline{a}\left(B ; \succ_{i}\right) \nsucc_{i}$ a for every $a \in B$.

We abbreviate $\bar{a}\left(A ; \succ_{i}\right), \underline{a}\left(A ; \succ_{i}\right)$ as $\bar{a}\left(\succ_{i}\right), \underline{a}\left(\succ_{i}\right)$ respectively. Given an alternative $a \in A$ and a profile $\succ_{N} \in \mathcal{L}^{N}$, let $S\left(a ; \succ_{N}\right)$ denote the set of agents, called supporters, whose best alternative under $\succ_{N}$ is a, i.e.,

$$
S\left(a ; \succ_{N}\right)=\left\{i \in N \mid a=\bar{a}\left(\succ_{i}\right)\right\}
$$

Also define $S^{C}\left(a ; \succ_{N}\right)=N-S\left(a ; \succ_{N}\right)$.

We first consider nonmanipulable restricted social choice functions(RSCFs). Each RSCF either attains an alternative in $A$ or produces no outcome according to profiles in $\mathcal{L}^{N}$. By $\mathcal{D}_{f}$ we denote the domain of an RSCF $f$. It is assumed that $\mathcal{D}_{f}$ is a nonempty subset of $\mathcal{L}^{N}$. An RSCF $f$ is formally defined as a mapping $f: \mathcal{D}_{f} \rightarrow A$. An RSCF $f$ is said to be nonmanipulable, if there exists no coalition $S$, profiles $\succ_{N}$ and $\succ_{N}^{\prime}=\left(\succ_{N-S}, \succ_{S}^{\prime}\right) \in \mathcal{D}_{f}$ such that $f\left(\succ_{N}^{\prime}\right) \succ_{i} f\left(\succ_{N}\right)$ for every $i \in S$.

By $\left|f\left(\mathcal{D}_{f}\right)\right|$ we denote the cardinality of its range. Let $\mathcal{F}$ be the class of all RSCFs that produce at least two distinct outcomes of $A$, i.e., $\mathcal{F}=\left\{f: \mathcal{D}_{f} \rightarrow A|| f\left(\mathcal{D}_{f}\right) \mid \geqq 2\right\}$.

We shall state a property of RSCF, which plays an important role through this paper.

Definition 1 An RSCF $f \in \mathcal{F}$ has the noncovering property(NC, for short) if $f$ satisfies the following condition:

$$
\begin{aligned}
& \text { For every } \succ_{N}^{1}, \succ_{N}^{2}, \cdots, \succ_{N}^{r} \in \mathcal{D}_{f} \text { and } \\
& \text { for every set of distinct alternatives } a_{1}, a_{2}, \ldots, a_{r}(1 \leqq r \leqq m) \text { such that } \\
& \qquad \begin{array}{l}
a_{j} \neq f\left(\succ_{N}^{j}\right) \text { for every } j=1,2, \cdots, r, \\
\bigcup_{j=1}^{r} S\left(a_{j} ; \succ_{N}^{j}\right) \neq N .
\end{array}
\end{aligned}
$$

This property means that any set $S\left(a ; \succ_{N}^{j}\right)$ such that $a \neq f\left(\succ_{N}^{j}\right)$ does not have relatively many agents.

Note that if $f$ satisfies NC, then $f\left(\succ_{N}\right) \neq a$ for every $\succ_{N} \in \mathcal{L}^{N}$ and $a \in A$ such that $S\left(a ; \succ_{N}\right)=\emptyset$.

Proposition 1 The NC condition is a sufficient condition for $f$ to be coalitionally nonmanipulable.

Proof : Suppose there exists a coalition $S$ and profiles $\succ_{N}$ and $\left(\succ_{N-S}, \succ_{S}^{\prime}\right)$ such that $f\left(\succ_{N-S}, \succ_{S}^{\prime}\right) \succ_{i} f\left(\succ_{N}\right)=a_{1}$ for every $i \in S$. Since $a_{1} \neq \bar{a}\left(\succ_{i}\right)$ for every $i \in S$, we have $S\left(a_{1} ;\left(\succ_{N-S}, \succ_{S}^{\prime}\right)\right) \supset S\left(a_{1} ; \succ_{j}\right)$. Set $\succ_{N}^{1}=\left(\succ_{N-S}, \succ_{S}^{\prime}\right)$ and $\succ_{N}^{j}=\succ_{N}$. for $j=2,3, \cdots, m$. Then we have $\bigcup_{j=1}^{m} S\left(a_{j} ; \succ_{N}^{j}\right)=S\left(a_{1} ;\left(\succ_{N-S}, \succ_{S}^{\prime}\right)\right) \cup \bigcup_{j=2}^{m} S\left(a_{j} ; \succ_{N}\right)=S\left(a_{1} ;\left(\succ_{N-S}, \succ_{S}^{\prime}\right.\right.$ )) $\cup S^{C}\left(a_{1} ; \succ_{N}\right)=N$, which means that $f$ does not satisfy NC.

Let $\mathcal{F}^{*}$ be the class of all RSCFs in $\mathcal{F}$ satisfying NC.

It should be noted that $\mathcal{F}^{*}$ is nonvacuous. The following example provides a simple subclass of $\mathcal{F}^{*}$. 
Example 1 Weighted Majority rules

$$
f\left(\succ_{N}\right)=a_{j} \Rightarrow \text { for every } k(\neq j) \in\{1,2, \cdots, m\}, \sum_{i \in S\left(a_{k} ; \succ_{N}\right)} p_{i}<q_{k}
$$

where $p_{1}, p_{2}, \ldots, p_{n}$ and $q_{1}, q_{2}, \ldots, q_{m}$ are positive coefficients assigned to the agents and alternatives respectively satisfying $\sum_{i=1}^{n} p_{i}=1$ and $\sum_{j=1}^{m} q_{j}=1$. Note that, we have $\emptyset \neq$ $\mathcal{D}_{f} \subset\left\{\succ_{N} \mid\right.$ for every $\left.k(\neq j) \in\{1,2, \cdots, m\}, \sum_{i \in S\left(a_{k} ; \succ_{N}\right)} p_{i}<q_{k}\right\}$.

Indeed, every $f$ satisfies NC condition. For every $\succ_{N}^{1}, \succ_{N}^{2}, \cdots, \succ_{N}^{r} \in \mathcal{D}_{f}$ and for every distinct alternative $a_{1}, a_{2}, \ldots, a_{r}(1 \leqq r \leqq m)$ such that $a_{j} \neq f\left(\succ_{N}^{j}\right)$ for every $j=1,2, \cdots, r$, we have $\sum_{i \in S\left(a_{j} ; \succ_{N}^{j}\right)} p_{i}<q_{j}$ for every $j=1,2, \cdots, r$. Summing up the inequalities for all $j=1,2, \cdots, r$, we get $\sum_{j=1}^{r} \sum_{i \in S\left(a_{j} ; \succ_{N}^{j}\right)} p_{i}<\sum_{j=1}^{r} q_{j} \leqq 1$. Since $\sum_{i \in \bigcup_{j=1}^{r} S\left(a_{j} ; \succ_{N}^{j}\right)} p_{i}$ $\leqq \sum_{j=1}^{r} \sum_{i \in S\left(a_{j} ; \succ_{N}^{j}\right)} p_{i}$, we have $\sum_{i \in \bigcup_{j=1}^{r} S\left(a_{j} ; \succ_{N}^{j}\right)} p_{i}<1$, which means that $\bigcup_{j=1}^{r} S\left(a_{j} ; \succ_{N}^{j}\right) \neq$ $N$.

When $p_{1}=p_{2}=\cdots=p_{n}=1 / n$ and $q_{1}=q_{2}=\cdots=q_{m}=1 / m$, the weighted majority rule becomes the $(m-1) / m$-majority rule, i.e.,

$$
f\left(\succ_{N}\right)=a \Rightarrow\left|S\left(a ; \succ_{N}\right)\right|>\frac{m-1}{m} n \text {. }
$$

Indeed, if $f\left(\succ_{N}\right)=a_{j}$ then for every alternative $a_{k} \neq a_{j}$, we have $\left|S\left(a_{k} ; \succ_{N}\right)\right|<\frac{1}{m} n$, that is, $\frac{1}{n}\left|S\left(a_{k} ; \succ_{N}\right)\right|<\frac{1}{m}=q_{k}$. Thus we get $\sum_{i \in S\left(a_{k} ; \succ_{N}\right)} p_{i}=\sum_{i \in S\left(a_{k} ; \succ_{N}\right)} \frac{1}{n}<q_{k}$, which is the condition of the weighted majority rule.

Finally, if $q_{1}=q_{2}=\cdots=q_{m}=1 / m, p_{i^{*}}=1-1 / m+1 / m n$ and $p_{i}=1 / m n$ for every $i \neq i^{*}$, then the agent $i^{*}$ is a dictator.

\subsection{Social choice correspondences}

A social choice correspondence $(\mathrm{SCC}) G$ is a mapping from $\mathcal{L}^{N}$ to $2^{A}$. When $G\left(\succ_{N}\right.$ ) $\neq \emptyset$ for every $\succ_{N} \in \mathcal{L}^{N}$, the SCC $G$ is said to be stable.

The most famous example of SCC is the core. In our context, the core is induced from $f$ as follows:

Definition 2 Let $f$ be an $R S C F$. The $S C C$ core is

$$
\begin{aligned}
C_{f}\left(\succ_{N}\right) \equiv\left\{a \in A \mid \text { for every } b(\neq a) \in A, S \subset\left\{i \in N \mid b \succ_{i} a\right\} \text { and } \succ_{S}^{\prime},\right. \\
\left.\left(\succ_{N-S}, \succ_{S}^{\prime}\right) \notin \mathcal{D}_{f} \text { or } f\left(\succ_{N-S}, \succ_{S}^{\prime}\right) \neq b\right\} .
\end{aligned}
$$

Now, we define the hypercore $H_{f}$ for a given RSCF $f$.

Definition 3 Let $f$ be an RSCF. An $S C C H_{f}$, called hypercore, is

$$
H_{f}\left(\succ_{N}\right) \equiv\left\{a \in A \mid \text { for every } \succ_{N}^{\prime} \in \mathcal{D}_{f} ; S\left(a ; \succ_{N}^{\prime}\right) \supset S\left(a ; \succ_{N}\right), f\left(\succ_{N}^{\prime}\right)=a\right\} \text {. }
$$

Example 2 Recall the weighted majority rule mentioned in Example 1. The hypercore $H_{f}$ of the RSCF $f$ corresponding to the weighted majority rule is as follows:

$$
H_{f}\left(\succ_{N}\right) \supset\left\{a_{j} \in A \mid \sum_{i \in S\left(a_{j} ; \succ_{N}\right)} p_{i} \geqq q_{j}\right\} \text {. }
$$

Because for every alternative $a_{j}$ such that $\sum_{i \in S\left(a_{j} ; \succ_{N}\right)} p_{i} \geqq q_{j}, S\left(a_{j} ; \succ_{N}^{\prime}\right) \supset S\left(a_{j} ; \succ_{N}\right)$ implies $\sum_{i \in S\left(a_{j} ; \succ_{N}^{\prime}\right)} p_{i} \geqq q_{j}$. It means that $\succ_{N}^{\prime} \notin \mathcal{D}_{f}$ or $f\left(\succ_{N}^{\prime}\right)=a_{j}$. Thus we have $a_{j} \in H_{f}\left(\succ_{N}\right)$.

If the weighted majority rule $f$ satisfies $f\left(\succ_{N}\right)=a_{j} \Leftrightarrow$ for every $k(\neq j) \in\{1,2, \cdots, m\}$, $\sum_{i \in S\left(a_{k} ; \succ_{N}\right)} p_{i}<q_{k}$, we have $H_{f}\left(\succ_{N}\right)=\left\{a_{j} \in A \mid \sum_{i \in S\left(a_{j} ; \succ_{N}\right)} p_{i} \geqq q_{j}\right\}$. Because for every alternative $a_{j}$ such that $\sum_{i \in S\left(a_{j} ; \succ_{N}\right)} p_{i}<q_{j}$, the following profile $\succ_{N}^{\prime} \in \mathcal{D}_{f}$ satisfies that $S\left(a_{j} ; \succ_{N}^{\prime}\right)=S\left(a_{j} ; \succ_{N}\right)$ and $f\left(\succ_{N}^{\prime}\right) \neq a_{j}$ :

$$
\succ_{N}^{\prime}= \begin{cases}\succ_{i}^{\prime}=\succ_{i} & \text { if } i \in S\left(a_{j} ; \succ_{N}\right) \\ \bar{a}\left(\succ_{i}^{\prime}\right)=a_{k}\left(\neq a_{j}\right) & \text { otherwise. }\end{cases}
$$

When the RSCF $f$ is the $(m-1) / m$-majority rule, the hypercore of $f$ is as follows:

$$
H_{f}\left(\succ_{N}\right) \supset\left\{a \in A|| S\left(a ; \succ_{N}\right) \mid \geqq \frac{1}{m}\right\} .
$$


The nonmanipulability based on max-min criterion for SCC is as follows:

Definition 4 A stable $S C C G$ is coalitionally nonmanipulable if for every $\succ_{N} \in \mathcal{L}^{N}$, there does not exist a coalition $S$, a profile $\succ_{N}^{\prime}=\left(\succ_{N-S}, \succ_{S}^{\prime}\right)$ and an alternative $a \in G\left(\succ_{N}\right)$ satisfying that

$a^{\prime} \succ_{i}$ a for every $a^{\prime} \in G\left(\succ_{N}^{\prime}\right)$ and every $i \in S$.

If an SCC $G$ is coalitionally manipulable, i.e., for some profile $\succ_{N}$, there exists an $a \in G\left(\succ_{N}\right)$ and $S, \succ_{S}^{\prime}$ satisfying that $a^{\prime} \succ_{i}$ a for every $a^{\prime} \in G\left(\succ_{N-S}, \succ_{S}^{\prime}\right)=G\left(\succ_{N}^{\prime}\right)$ and $i \in S$, we say that the coalition $S$ can manipulate $G\left(\succ_{N}\right)$ through the strategy $\succ_{S}^{\prime}$. Since the alternative $a$ satisfies $a \succ_{i} \underline{a}\left(G\left(\succ_{N}\right) ; \succ_{i}\right)$ or $a=\underline{a}\left(G\left(\succ_{N}\right) ; \succ_{i}\right)$, we have $\underline{a}\left(G\left(\succ_{N}^{\prime}\right) ; \succ_{i}\right) \succ_{i} \underline{a}\left(G\left(\succ_{N}\right) ; \succ_{i}\right)$ for every $i \in S$. This fact implies that if $S$ can manipulate $G\left(\succ_{N}\right)$ through $\succ_{S}^{\prime}$ then for each member of $S, G\left(\succ_{N}^{\prime}\right)$ is more preferable than $G\left(\succ_{N}\right)$ in the max-min sense. On the contrary, we have $\bar{a}\left(G\left(\succ_{N}^{\prime}\right) ; \succ_{i}\right) \succ_{i} \bar{a}\left(G\left(\succ_{N}\right) ; \succ_{i}\right)$ for every $i \in S$ if the coalition $S$ can manipulate the SCC $G$ through the strategy $\succ_{S}^{\prime}$ in DEMANGE's definition [4] which is based on the max-max sense.

Next examples demonstrate the manipulability of the core and the nonmanipulability of the hypercore in the max-min sense.

Example 3 Consider the 3/4-majority rule with $N=\{1,2, \cdots, 5\}$ and $A=\{a, b, c, d\}$. Set the profile $\succ_{N}$ and $\succ_{\{1,2\}}^{\prime}$ as follows:

$$
\begin{aligned}
& a \succ_{1} b \succ_{1} c \succ_{1} d, a \succ_{2} b \succ_{2} d \succ_{2} c, a \succ_{3} c \succ_{3} b \succ_{3} d, b \succ_{4} d \succ_{4} a \succ_{4} c, \\
& d \succ_{5} c \succ_{5} b \succ_{5} a \text { and } a \succ_{1}^{\prime} c \succ_{1}^{\prime} b \succ_{1}^{\prime} d, a \succ_{2}^{\prime} c \succ_{2}^{\prime} b \succ_{2}^{\prime} d .
\end{aligned}
$$

Then we have $C_{f}\left(\succ_{N}\right)=\{a, b\}$ and $C_{f}\left(\succ_{\{1,2\}}^{\prime}, \succ_{\{3,4,5\}}\right)=\{a\}$, that is, the coalition $\{1,2\}$ can manipulate the core through the strategy $\succ_{\{1,2\}}^{\prime}$. Thus the core is manipulable in our context as this example shows. On the contrary, we have $H_{f}\left(\succ_{N}\right)=\{a\}$ and $H_{f}\left(\succ_{\{1,2\}}^{\prime}, \succ_{\{3,4,5\}}\right)=$ $\{a\}$. This example suggests the hypercore of this example can not be manipulated by any coalition.

When the agent 5 uses the strategy $b \succ_{5}^{\prime \prime} d \succ_{5}^{\prime \prime} c \succ_{5}^{\prime \prime} a$, we have $H_{f}\left(\succ_{\{1,2,3,4\}}, \succ_{5}^{\prime \prime}\right)=\{a, b\}$. Since $b=\bar{a}\left(\{a, b\} ; \succ_{5}\right) \succ_{5} \bar{a}\left(\{a\} ; \succ_{5}\right)=a$, the agent 5 can manipulate the hypercore in the max-max sense. However, any coalition can not manipulate the core in the max-max sense. If the coalition $S$ can manipulate $C_{f}\left(\succ_{N}\right)$ in the max-max sense, then it must satisfy $|S| \geqq 4$ and there must exist an alternative $x \notin C_{f}\left(\succ_{N}\right)$ such that $x \succ_{i} y$ for every $i \in S$, where $y=\bar{a}\left(C_{f}\left(\succ_{N}\right) ; \succ_{i}\right)$ for every $i \in S$. By the definition of the core, we have $y \notin C_{f}\left(\succ_{N}\right)$ which is a contradiction.

\section{Properties of the Hypercore}

From the definition of the hypercore, we can immediately see that the hypercore is included in the core for every profile. Moreover, we can see the following properties of the hypercore.

Lemma 1 Let $f$ be an RSCF. If $f\left(\succ_{N}\right)=a$ then $H_{f}\left(\succ_{N}\right) \subset\{a\}$.

Proof : For every $b \neq a, f\left(\succ_{N}\right)=f\left(\succ_{S\left(b ; \succ_{N}\right)}, \succ_{S^{C}\left(b ; \succ_{N}\right)}\right)=a \neq b$. From the definition of $H_{f}\left(\succ_{N}\right), b \notin H_{f}\left(\succ_{N}\right)$.

This property expresses when a profile $\succ_{N}$ belongs to the domain of a given RSCF $f$, the hypercore $H_{f}$ of the profile does not include any alternative except $f\left(\succ_{N}\right)$.

Lemma 2 Let $f \in \mathcal{F}$. If $S\left(a ; \succ_{N}\right)=\emptyset$ then $a \notin H_{f}\left(\succ_{N}\right)$.

Proof : We see that for every $\succ_{N}^{\prime}, S\left(a ; \succ_{N}^{\prime}\right) \supset S\left(a ; \succ_{N}\right)$. Since $\left|f\left(\mathcal{D}_{f}\right)\right| \geqq 2$, there exists $\succ_{N}^{\prime}$ such that $f\left(\succ_{N}^{\prime}\right)=b \neq a$. From the definition of $H_{f}, a \notin H_{f}\left(\succ_{N}\right)$.

This lemma means that if an alternative has no supporter at a profile, then the alternative is not included in the hypercore for any $f$ at the profile.

We consider the monotonicity of SCC with respect to supporters. 
Definition 5 An SCC $G$ is said to be monotonic, if $a \in G\left(\succ_{N}^{\prime}\right)$ for every $\succ_{N}^{\prime} \in \mathcal{L}^{N}$ such that $S\left(a ; \succ_{N}^{\prime}\right) \supset S\left(a ; \succ_{N}\right)$ whenever $a \in G\left(\succ_{N}\right)$.

Lemma 3 Let $f$ be an $R S C F$. The hypercore $H_{f}$ is monotonic.

Proof : Since $a \in H_{f}\left(\succ_{N}\right)$, for every $\succ_{N}^{\prime \prime}$ such that $S\left(a ; \succ_{N}^{\prime \prime}\right) \supset S\left(a ; \succ_{N}^{\prime}\right)$, we have $f\left(\succ_{N}^{\prime \prime}\right.$ ) $=a$ or $\succ_{N}^{\prime \prime} \notin \mathcal{D}_{f}$. Hence $a \in H_{f}\left(\succ_{N}^{\prime}\right)$.

Lemma 4 Let $f$ be an $R S C F$. If a $\notin H_{f}\left(\succ_{N}\right)$ then a $\notin H_{f}\left(\succ_{N}^{\prime}\right)$ for every $\succ_{N}^{\prime} \in \mathcal{L}^{N}$ such that $S\left(a ; \succ_{N}^{\prime}\right) \subset S\left(\alpha ; \succ_{N}\right)$.

Proof : As $a \notin H_{f}\left(\succ_{N}\right)$, there exists $\succ_{N}^{\prime \prime}$ such that $S\left(a ; \succ_{N}^{\prime \prime}\right) \supset S\left(a ; \succ_{N}\right)$ and $f\left(\succ_{N}^{\prime \prime}\right)=b \neq$ a. $S\left(a ; \succ_{N}^{\prime}\right) \subset S\left(a ; \succ_{N}\right)$ implies that $S\left(a ; \succ_{N}^{\prime \prime}\right) \supset S\left(a ; \succ_{N}^{\prime}\right)$. Hence $a \notin H_{f}\left(\succ_{N}^{\prime}\right)$.

Note that Lemma 4 means that $H_{f}$ is monotonic with respect to the decrease in supporters.

Lemma 5 Let $f$ be an RSCF. For every profiles $\succ_{N}, \succ_{N}^{\prime} \in \mathcal{L}^{N}$ such that $S\left(a ; \succ_{N}\right)=$ $S\left(a ; \succ_{N}^{\prime}\right)$ for every $a \in A, H_{f}\left(\succ_{N}\right)=H_{f}\left(\succ_{N}^{\prime}\right)$.

Proof : For every $a \in H_{f}\left(\succ_{N}\right)$, since $S\left(a ; \succ_{N}^{\prime}\right) \supset S\left(a ; \succ_{N}\right)$, we have $a \in H_{f}\left(\succ_{N}^{\prime}\right)$ from Lemma 3. For every $a \notin H_{f}\left(\succ_{N}\right)$, since $S\left(a ; \succ_{N}^{\prime}\right) \subset S\left(a ; \succ_{N}\right)$, we have $a \notin H_{f}\left(\succ_{N}^{\prime}\right)$ from Lemma 4.

This property enable us to divide the profiles into equivalent classes according to the values of hypercore.

Moreover, we can see the following relation between the core and the hypercore. The name hypercore stems from following result.

Theorem 1 For every profile $\succ_{N} \in \mathcal{L}^{N}, H_{f}\left(\succ_{N}\right)$ coincides with the intersection of $C_{f}\left(\succ_{N}^{\prime}\right)$ over all profiles $\succ_{N}^{\prime} \in \mathcal{L}^{N}$ such that $S\left(a ; \succ_{N}^{\prime}\right)=S\left(a ; \succ_{N}\right)$ for every $a \in A$. That is, for every profile $\succ_{N} \in \mathcal{L}^{N}$, we have the following equation:

$$
H_{f}\left(\succ_{N}\right)=\bigcap_{S\left(\bullet ; \succ_{N}^{\prime}\right)=S\left(\bullet ; \succ_{N}\right)} C_{f}\left(\succ_{N}^{\prime}\right) \text {. }
$$

Proof : For every $\succ_{N} \in \mathcal{L}^{N}$, we have $\left\{i \in N \mid b \succ_{i} a\right\} \subset S^{C}\left(a ; \succ_{N}\right)$ for every alternative $b(\neq a) \in A$. That is, for every $b(\neq a) \in A, S \subset\left\{i \in N \mid b \succ_{i} a\right\}$ and $\succ_{N}^{\prime}=\left(\succ_{N-S}, \succ_{S}^{\prime}\right)$, we have $S\left(a ; \succ_{N}^{\prime}\right) \supset S\left(a ; \succ_{N}\right)$.

If $a \in H_{f}\left(\succ_{N}\right)$ holds, then, from the definition of the hypercore, for every $b(\neq a) \in A$, $S \subset\left\{i \in N \mid b \succ_{i} a\right\}$ and $\succ_{N}^{\prime}=\left(\succ_{N-S}, \succ_{S}^{\prime}\right)$, we have $\succ_{N}^{\prime} \notin \mathcal{D}_{f}$ or $f\left(\succ_{N}^{\prime}\right)=a$. Thus $a \in C_{f}\left(\succ_{N}\right)$.

Since $a \in H_{f}\left(\succ_{N}^{\prime}\right)$ holds from Lemma 5 , for every $\succ_{N}^{\prime} \in \mathcal{L}^{N}$ such that $S\left(\bullet ; \succ_{N}^{\prime}\right)=S\left(\bullet ; \succ_{N}\right.$ ), we have $a \in C_{f}\left(\succ_{N}^{\prime}\right)$. Thus we have $H_{f}\left(\succ_{N}\right) \subset C_{f}\left(\succ_{N}^{\prime}\right)$ for every $\succ_{N}^{\prime} \in \mathcal{L}^{N}$ satisfying $S\left(\bullet ; \succ_{N}^{\prime}\right)=S\left(\bullet ; \succ_{N}\right)$.

On the other hand, if $a \notin H_{f}\left(\succ_{N}\right)$ holds, then there exists a profile $\succ_{N}^{\prime} \in \mathcal{D}_{f}$ such that $S\left(a ; \succ_{N}^{\prime}\right) \supset S\left(a ; \succ_{N}\right)$ and $f\left(\succ_{N}^{\prime}\right)=b \neq a$.

For every $i \in S^{C}\left(a ; \succ_{N}\right)=T$, choose a preference ordering $\succ_{i}^{\prime \prime}$ satisfying $\underline{a}\left(\succ_{i}^{\prime \prime}\right)=a$ and $\bar{a}\left(\succ_{i}^{\prime \prime}\right)=\bar{a}\left(\succ_{i}\right)$. Then the profile $\succ_{N}^{\prime \prime}=\left(\succ_{T^{C}}^{\prime}, \succ_{T}^{\prime \prime}\right)$ satisfies $S\left(\bullet ; \succ_{N}^{\prime \prime}\right)=S\left(\bullet ; \succ_{N}\right)$. Furthermore, since there exists a coalition $\left\{i \in N \mid b \succ_{i}^{\prime \prime} a\right\}=S^{C}\left(a ; \succ_{N}\right)=T$ such that $f\left(\succ_{T^{C}}^{\prime \prime}, \succ_{T}^{\prime}\right)=f\left(\succ_{N}^{\prime}\right)=b$, we have $a \notin C_{f}\left(\succ_{N}^{\prime \prime}\right)$.

Next example demonstrates this property.

Example 4 Consider the 2/3-majority rule with $N=\{1,2,3,4\}$ and $A=\{a, b, c\}$. Define $\succ_{N}$ and $\succ_{N}^{\prime}$ by

$a \succ_{1} b \succ_{1} c, a \succ_{2} b \succ_{2} c, b \succ_{3} c \succ_{3} a, c \succ_{4} b \succ_{4} a$ and

$a \succ_{i}^{\prime} c \succ_{i}^{\prime} b, i=1,2, \succ_{j}^{\prime}=\succ_{j}, j=3,4$.

Then we have $\succ_{N}, \succ_{N}^{\prime} \notin \mathcal{D}_{f}, C_{f}\left(\succ_{N}\right)=\{a, b\}, C_{f}\left(\succ_{N}^{\prime}\right)=\{a, c\}$ and $H_{f}\left(\succ_{N}\right)=H_{f}\left(\succ_{N}^{\prime}\right)=$ $\{a\} \subset C_{f}\left(\succ_{N}\right) \cap C_{f}\left(\succ_{N}^{\prime}\right)$.

Next, we consider the relation between the domain of RSCF $f$ and the value of the hypercore $H_{f}$. 
Proposition 2 Let $f, f^{\prime}$ be RSCFs, and $\mathcal{D}_{f}, \mathcal{D}_{f^{\prime}}$ be the domains of $f, f^{\prime}$ such that $\mathcal{D}_{f} \supset \mathcal{D}_{f^{\prime}}$ and $f\left(\succ_{N}\right)=f^{\prime}\left(\succ_{N}\right)$ for every $\succ_{N} \in \mathcal{D}_{f^{\prime}}$. Then $H_{f}\left(\succ_{N}\right) \subset H_{f^{\prime}}\left(\succ_{N}\right)$ for every $\succ_{N} \in \mathcal{L}^{N}$.

Proof : From the definition of $H_{f}$, for each $a \in H_{f}\left(\succ_{N}\right), f\left(\succ_{N}^{\prime}\right)=a$ for every $\succ_{N}^{\prime} \in \mathcal{D}_{f}$ such that $S\left(a ; \succ_{N}^{\prime}\right) \supset S\left(a ; \succ_{N}\right)$. From the condition of $f$ and $f^{\prime}, \succ_{N} \notin \mathcal{D}_{f}$ implies $\succ_{N} \notin \mathcal{D}_{f^{\prime}}$ and $f\left(\succ_{N}\right)=a$ implies $f^{\prime}\left(\succ_{N}\right)=a$ or $\succ_{N} \notin \mathcal{D}_{f^{\prime}}$. Thus we have $a \in H_{f^{\prime}}\left(\succ_{N}\right)$.

Recall the weighted majority rule mentioned in Example 1 again. If the weighted majority rule $f$ satisfies $f\left(\succ_{N}\right)=a_{j} \Leftrightarrow$ for every $k(\neq j) \in\{1,2, \cdots, m\}, \sum_{i \in S\left(a_{k} ; \succ_{N}\right)} p_{i}<q_{k}$, then $f$ has the maximal domain among the rules having the same coefficients. Since $f$ has the maximum domain, the value of the hypercore $H_{f}$ becomes minimal set. Thus $H_{f}$ is exactly defined in Example 2.

For some $H_{f}$, if there exists a profile $\succ_{N}$ such that $\left|H_{f}\left(\succ_{N}\right)\right| \geqq 2$, then $H_{f}\left(\succ_{N}\right) \ni a \neq$ $\bar{a}\left(\succ_{i}\right)$ for each $i \in N$. Therefore, such $H_{f}$ satisfies weak nondictatorialness. Let us now discuss stronger nondictatorialness. We say the RSCF $f$ is nondictatorial if for every agent $i \in N$, there exists a profile $\succ_{N}^{i} \in \mathcal{D}_{f}$ such that $f\left(\succ_{N}^{i}\right) \neq \bar{a}\left(\succ_{i}^{i}\right)$. Furthermore, we say the SCC $G$ is nondictatorial if for every agent $i \in N$, there exists a profile $\succ_{N}^{i} \in \mathcal{L}^{N}$ such that $G\left(\succ_{N}^{i}\right) \not \supset \bar{a}\left(\succ_{i}^{i}\right)$.

Proposition 3 An $R S C F f \in \mathcal{F}$ is nondictatorial if and only if $H_{f}$ is nondictatorial.

Proof: From Lemma 1, $f\left(\succ_{N}\right) \neq a$ implies $H_{f}\left(\succ_{N}\right) \subset A-\{a\}$. Thus, if $f$ is nondictatorial, then for every $i \in N$, there exists a profile $\succ_{N}^{i} \in \mathcal{D}_{f}$ such that $H_{f}\left(\succ_{N}^{i}\right) \not \supset \bar{a}\left(\succ_{i}^{i}\right)$.

From the definition of the hypercore, if $H_{f}\left(\succ_{N}\right) \not \supset a$ then there exists a profile $\succ_{N}^{\prime} \in \mathcal{D}_{f}$ such that $f\left(\succ_{N}^{\prime}\right) \neq a$. Thus, if $H_{f}$ is nondictatorial, then for every $i \in N$, there exists $\succ_{N}^{i} \in \mathcal{D}_{f}$ such that $f\left(\succ_{N}^{i}\right) \neq \bar{a}\left(\succ_{i}^{i}\right)$.

Furthermore, we can see a theorem that claim the stability of hypercore.

Theorem 2 An $R S C F f \in \mathcal{F}$ belongs to $\mathcal{F}^{*}$ if and only if $H_{f}$ is stable.

Proof :

(Necessity.) Suppose that there is a preference profile $\succ_{N} \in \mathcal{L}^{N}$ such that $H_{f}\left(\succ_{N}\right)=\emptyset$. Then for each $a_{j}, j=1,2, \cdots, m$, there exists $\succ_{N}^{j}$ such that $f\left(\succ_{N}^{j}\right)=b_{j} \neq a_{j}$ and $S\left(a_{j} ; \succ_{N}^{j}\right.$ ) つ $S\left(a_{j} ; \succ_{N}\right)$. Since $\left\{S\left(a_{j} ; \succ_{N}\right)\right\}_{j=1,2, \cdots, m}$ is a partition of $N, \cup_{j=1}^{m} S\left(a_{j} ; \succ_{N}^{j}\right)=N$. Thus $f$ does not satisfy NC.

(Sufficiency.) Suppose that $f$ does not satisfy NC. Then, without loss of generality, there exists $\succ_{N}^{1}, \succ_{N}^{2}, \cdots, \succ_{N}^{r}$ such that $f\left(\succ_{N}^{j}\right)=b_{j} \neq a_{j}$ and $\bigcup_{j=1}^{r} S\left(a_{j} ; \succ_{N}^{j}\right)=N$. Set $S_{j} \equiv$ $S\left(a_{j} ; \succ_{N}^{j}\right)-\bigcup_{k=1}^{j-1} S\left(a_{k} ; \succ_{N}^{k}\right), j=1,2, \cdots, r$, then $\left\{S_{j}\right\}_{j=1,2, \cdots, r}$ is a partition of $N$. For each $i \in N$, choose a preference ordering $\succ_{i}$ satisfying $\bar{a}\left(\succ_{i}\right)=a_{j}$ if and only if $S_{j} \ni i$. Now, suppose that there exists an $a_{l} \in H_{f}\left(\succ_{N}\right)$. Then we have $l \leqq r$, since, by Lemma 2, $S\left(a_{l} ; \succ_{N}\right)=\emptyset$ implies $a_{l} \notin H_{f}\left(\succ_{N}\right)$. From the definition of $H_{f}$, for every $\succ_{N}^{\prime}$ such that $S\left(a_{l} ; \succ_{N}^{\prime}\right) \supset S\left(a_{l} ; \succ_{N}\right)=S_{l}$, we have $f\left(\succ_{N}^{\prime}\right)=a_{l}$ or $\succ_{N}^{\prime} \notin \mathcal{D}_{f}$. As $S\left(a_{l} ; \succ_{N}^{l}\right) \supset S_{l}$, we have $f\left(\succ_{N}^{l}\right)=a_{l}$ or $\succ_{N}^{l} \notin \mathcal{D}_{f}$. However, from the assumption on $f, f\left(\succ_{N}^{l}\right)=b_{l} \neq a_{l}$, which is a contradiction. Therefore $H_{f}\left(\succ_{N}\right)=\emptyset$.

If an RSCF $f$ satisfies NC, then we obtain the following property of the hypercore.

Proposition 4 Let $f \in \mathcal{F}^{*}$. If $f\left(\succ_{N}\right)=a$ then $H_{f}\left(\succ_{N}\right)=\{a\}$.

Proof : From Lemma 1 we have $H_{f}\left(\succ_{N}\right) \subset\{a\}$. Since $f$ satisfies NC, from Theorem 2, the hypercore is stable. It follows that $H_{f}\left(\succ_{N}\right)=\{a\}$.

Theorem 3 Let $G$ be an $S C C$. If $G$ is monotonic then $G$ is coalitionally nonmanipulable.

Proof: Suppose this is false. Let $S$ be a coalition which can manipulate $G\left(\succ_{N}\right)$ through $\succ_{N}^{\prime}$, i.e., there exists profiles $\succ_{N}, \succ_{N}^{\prime}=\left(\succ_{N-S}, \succ_{S}^{\prime}\right)$, an alternative $a \in G\left(\succ_{N}\right)$ such that $a^{\prime} \succ_{i} a$ for every $a^{\prime} \in G\left(\succ_{N}^{\prime}\right)$ and every $i \in S$.

Since $a \notin G\left(\succ_{N}^{\prime}\right), a \neq \bar{a}\left(\succ_{i}\right)$ for any $i \in S$. This implies $S \subset S^{C}\left(a ; \succ_{N}\right)$, that is, 
$S\left(a ; \succ_{N}^{\prime}\right) \supset S\left(a ; \succ_{N}\right)$. Since $G$ is monotonic, we have $a \in G\left(\succ_{N}^{\prime}\right)$. This is a contradiction.

Proposition 5 For every $f \in \mathcal{F}, H_{f}$ is coalitionally nonmanipulable.

Proof: As mentioned in Lemma $3, H_{f}$ is monotonic. Thus $H_{f}$ is coalitionally nonmanipulable.

\section{Concluding Remarks}

We obtained a class of SCCs each of which is stable and coalitionally nonmanipulable by introducing the hypercore.

Once an RSCF $f$ is given, we can easily construct the hypercore $H_{f}$ by a simple polynomial time algorithm. Furthermore, to know whether or not a given RSCF $f$ satisfies the NC condition, it suffices to check the stability of $H_{f}$.

It is clear that the smaller cardinality of the value an SCC has, the better it behaves. Indeed, if the value of an SCC is the set $A$ itself for every profile, then the SCC is evidently nonmanipulable in any sense but it is meaningless. If we choose an RSCF having the maximal domain, we obtain 'minimal hypercore' according to Proposition 2. This situation is indicated in Examples 1 and 2.

\section{References}

[1] K.J.Arrow: Social choice and individual values, 2nd edition (Wiley, New York, 1962).

[2] S.Barbera, H.Sonnenschein and L.Zhou: Voting by committees. Econometrica, 59 (1991) 595-609.

[3] V.I.Danilov and A.I.Sotskov: On strongly consistent social choice functions. Journal of Mathematical Economics, 22 (1993) 327-346.

[4] G.Demange: Nonmanipulable cores. Econometrica, 55 (1987) 1057-1074.

[5] A.Gibbard: Manipulation of voting schemes: A general result. Econometrica, 41 (1973) 587-601.

[6] H.Moulin and B.Peleg: Cores of effectivity functions and implementation theory. Journal of Mathematical Economics, 10 (1982) 115-145.

[7] B.Peleg: Game theoretic analysis of voting in committees (Cambridge University Press, Cambridge, 1983).

[8] M.A.Satterthwaite: The existence of a strategy-Proof voting procedure: A topic in social choice theory (Ph.D. Dissertation, University of Wisconsin, Madison, 1973).

Masayoshi MIZUTANI:

Department of Business Administration, Tokyo Keizai University,

Kokubunji, Tokyo, 185-8502 JAPAN

E-mail: mizu@tku.ac.jp 